EDITORIAL

\title{
Trabajando en «tres turnos» en tiempos de pandemia: profesoras de Derecho y carrera académica en América Latina
}

\author{
María Francisca Elgueta \\ Directora
}

En el contexto de persistencia de la pandemia, nos pareció de enorme relevancia dar a conocer un primer avance de un estudio de casos, cuyo objetivo central es informar sobre el impacto de la pandemia en la carrera académica de profesoras de Derecho latinoamericanas.

Si con anterioridad a la pandemia la mujer trabajadora había avanzado en el logro de diferenciar el trabajo académico del trabajo de la casa, se vive un momento de retroceso en la medida en que la economía del cuidado (Flores-Sequera, 2020: 97) se ha vuelto más patente y no por ello más criticada. Se espera que las mujeres se desempeñen competentemente como académicas y que ejerzan labores tradicionales femeninas de género en las situaciones de encierro como «dueñas de casa», todo ello a pesar del exigente contexto de pandemia. Entonces, nos preguntamos cómo está afectando la pandemia la carrera académica de las profesoras latinoamericanas, en qué medida los fenómenos que tradicionalmente las han afectado en el desarrollo de su vida profesional se han visto potenciados y afectado negativamente su desempeño laboral, y en qué medida la gestión institucional admite la existencia de este escenario desfavorable y realiza acciones remediales en favor de las profesoras.

«Quédate en casa» (Flores-Sequera, 2020: 99) remite no solo al quehacer propio de la universidad, sino que al espacio del hogar en el que se configura una trama de relaciones en torno a las responsabilidades de familia y el trabajo doméstico, en el que se triplican las labores en comparación con la de sus pares masculinos (Cepal, 2020). Todo esto, en un contexto institucional en el que las representaciones de lo femenino y lo masculino responden a los discursos de la cultura dominante androcéntrica extracadémica (Fardella y otros, 2021: 4, Díaz, 2020).

Las profesoras se encuentran en contextos universitarios en los que, muchas veces, existe una falta de conciliación entre la vida universitaria y la vida personal, profundizándose un androcentrismo en las actividades de investigación (Díaz, 2020). Este fenómeno se ha agravado porque, además, se debe realizar una docencia remota 
virtual, en la que se han tenido que fortalecer las competencias digitales utilizando recursos que, idealmente, deben ser entregados por las universidades, algo que no está ocurriendo en toda Latinoamérica (Said-Hung y otros, 2021: 300).

En este contexto de pandemia, las profesoras están viviendo efectos en su salud física. Muchas tienen dolores de columna, de cabeza, anemia, desajustes del sueño, tendinitis y cansancio visual. No obstante, también «las secuelas psicológicas generadas por la incertidumbre y el entrecruzamiento de condiciones desfavorables en la vida personal y profesional son más severas en las mujeres» (Flores-Sequera, 2020: 95).

No obstante, de manera general, las profesoras mencionan: «la academia no es algo circunstancial, yo sé que la vida académica es lo que yo quiero hacer el resto de mi vida» (entrevistada 4). Por eso, se necesitan compromisos para implementar «políticas contracíclicas sensibles a las desigualdades de género [y] diseñar sistemas integrales de cuidado desde una perspectiva de género» (Cepal, 2020: 2). En la misma línea, es necesario abrir las oportunidades y fortalecer procesos en el que las políticas, los reglamentos, las culturas y tanto las prácticas institucionales como personales promuevan una mayor equidad.

Las profesoras que participaron del estudio compartieron la manera en la que han desarrollado, en el tiempo, sus carreras académicas, las situaciones de discriminación, las brechas en espacios masculinizados y cómo experimentan la «frontera de cristal» o aquella dicotomía entre vida familiar y trabajo (Burin, 2008: 83) en una situación de pandemia. La forma en la que han vivenciado la docencia remota de emergencia, sus investigaciones y las consecuencias del encierro en su salud mental y física, se complejizan cuando construyen sus subjetividades en torno a los modos de desear, sentir y pensar.

Las profesoras están experimentado un límite invisible u «opción de hierro», que se configura entre desarrollar las actividades propias de la academia que generan satisfacción o la necesidad de sostener vínculos familiares que también lo hacen (Burin, 2008: 83). Esta tensión entre mandatos laborales y mandatos culturales, referidos a la mano invisible que mantiene la producción social y prepara la producción dejando a la mujer con un rol de contención y cuidado, genera un evidente desgaste cotidiano que, unido a la incertidumbre de futuro que trae la pandemia, implican un riesgo de salud mental. A esto, se suma el teletrabajo, que involucra un aumento en las horas productivas en relación con el trabajo presencial (Flores-Sequera, 2020: 107).

Existe, de forma estructural en la carrera académica de las profesoras, una invisibilidad en torno a los límites posibles a alcanzar en puestos de liderazgo, lo que la literatura denomina «techo de cristal». Este concepto se traduce en límites invisibles para el acceso a puestos directivos en el que existen barreras implícitas, que si fueran explícitas serían ilegales. Esto genera una inequidad, ya que fortalece espacios masculinizados (Lara, 2007; Lozano y otros, 2016, Santos y otros, 2020). 


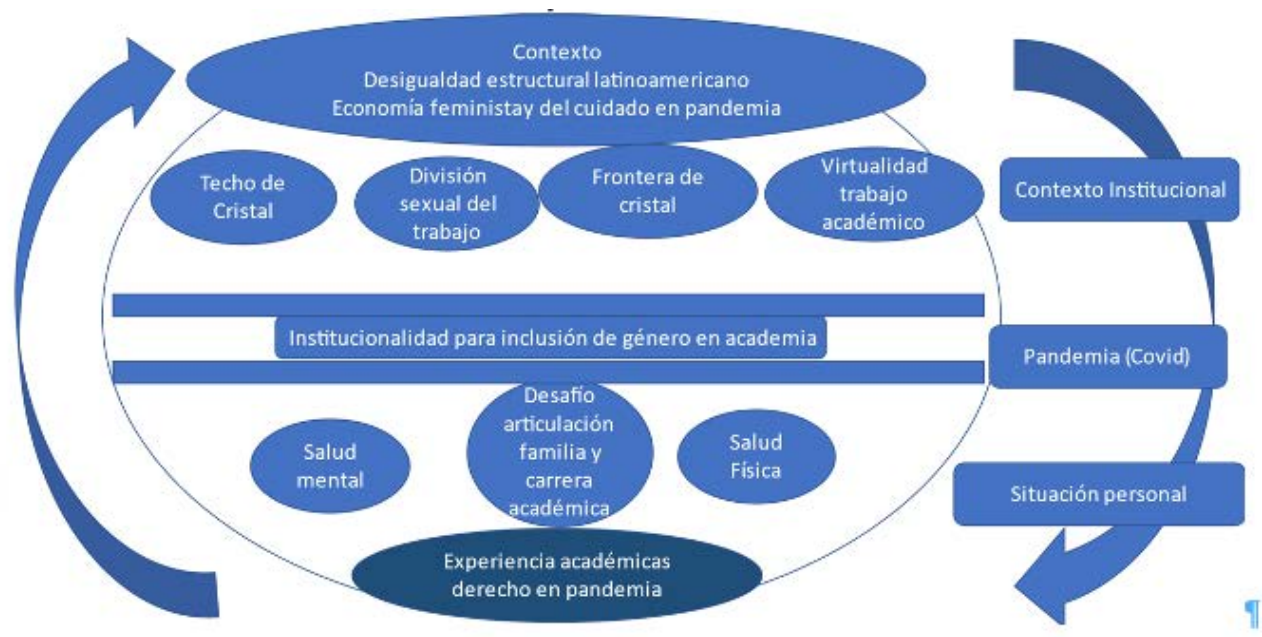

Figura 1. Oportunidades de desarrollo académico frenado por obstáculos estructurales en pandemia.

Los espacios masculinizados operan con una cultura en la que la mujer tiende a guardar silencio y aceptar la situación creyendo que, con sus méritos personales y esfuerzo, podrá lograr superar las dificultades para desarrollar investigación o docencia de manera competitiva. El tiempo muchas veces juega en contra, más aún cuando se está en una situación de pandemia, donde la salud mental se ve afectada por el aislamiento (García-González y otros, 2020: 309).

A medida de que las carreras académicas pasan de novel a etapa intermedia y fase senior, se van agudizando dificultades. Al comenzar, las profesoras creen que lograrán superar los desafíos al igual que sus colegas varones, sin embargo, a medida que avanzan, las opciones de maternidad y/o cuidado repercuten en una menor dedicación temporal a la academia, lo que queda en evidencia ya en la etapa senior, debido a que «la promoción en la academia se ve supeditada al género, pues las mujeres se ven enfrentadas a la disyuntiva de proyectar su carrera o privilegiar la familia» (Baeza y Lamadrid, 2019: 12). En la figura 1, se pueden apreciar las oportunidades de desarrollo académico frenado por obstáculos estructurales en este contexto.

Para abordar la problemática, realizamos un estudio de caso de tipo cualitativo (Stake, 2007) a profesoras de la carrera de Derecho de instituciones latinoamericanas de Brasil, Argentina, Colombia, Venezuela, Chile, Uruguay y México.

Es un estudio de caso porque «es algo específico, complejo y en funcionamiento» (Stake, 2007: 16). Su singularidad se expresa en la coherencia interna que da cuenta de aspectos en común y diferenciadores. Los factores en común hacen referencia a que: a) son todas profesoras con identidad de género femenino; b) imparten docencia en pregrado de derecho; c) están sometidas a la situación de pandemia en encierro; y d) viven en Latinoamérica. 
El estudio permite conocer la situación de las profesoras de Derecho en un mismo marco temporal de pandemia (2020-2021), pero considera las experiencias diferenciadas de cada una en relación con: a) los contextos socioeconómicos y políticos de cada país; b) la docencia en las carreras en pandemia; c) el momento de la carrera académica (novel, mitad del proceso, senior); y d) los reglamentos y la cultura institucional.

Se realizaron entrevistas individuales en profundidad (Taylor y Bogdan, 1984) con el propósito de comprender la experiencia académica de las profesoras y los efectos de la pandemia. El estudio se caracteriza por ser en profundidad y seguir la metodología de Stake (2007) a través del levantamiento de preguntas emergentes:

- En relación con el contexto de pandemia, ¿cómo han experimentado la pandemia en sus países?

- ¿Cómo han asumido la continuidad de la docencia en sus instituciones y de qué manera ha impactado esto en el trabajo académico?

- ¿Cómo han experimentado la virtualidad en sus trabajos académicos?

- En relación con sus carreras académicas, ¿han percibido situaciones de discriminación de género?

- ¿Han tenido puestos directivos?

- ¿En qué fase de sus carreras académicas están?

- ¿Cuánta dificultad han tenido para hacer sus carreras académicas?

- ¿La situación personal y familiar ha tenido alguna influencia en el desarrollo de sus carreras académicas?

En el aspecto referido a las características de las profesoras que participan del estudio de caso, están en diversas etapas de sus carreras académicas, trabajan en universidades públicas o privadas, poseen posgrados y pueden desarrollar con mayor énfasis docencia y/o investigación y/o gestión.

El contexto latinoamericano en el que se desempeñan las profesoras, se caracteriza por poseer una alta desigualdad y un lento crecimiento económico, siendo una de las zonas más violentas del mundo y existiendo relación entre violencia, desigualdad y productividad. Una manera de explicar la manera en la que esto ocurre es que existe una concentración del poder unido a violencia política, criminal y social, en el que no hay buenos diseños de protección social y claros marcos regulatorios de los mercados laborales, lo que distorsiona la economía. A esto, se suman las demandas de mayor justicia y la necesidad de reformas deseables en relación con formas locales de gobernanza o (in)gobernanza (PNUD, 2021: 2). 
Tabla 1. Características de las académicas que participan de la investigación

\begin{tabular}{|lllllll}
\hline País & $\begin{array}{l}\text { Tipo de } \\
\text { universidad }\end{array}$ & $\begin{array}{l}\text { Momento } \\
\text { carrera } \\
\text { académica }\end{array}$ & $\begin{array}{l}\text { Situación } \\
\text { remuneración } \\
\text { en pandemia }\end{array}$ & $\begin{array}{l}\text { Máximo grado } \\
\text { académico }\end{array}$ & $\begin{array}{l}\text { Reglamentos } \\
\text { género }\end{array}$ & $\begin{array}{l}\text { Preferencia, docencia, } \\
\text { investigación, gestión }\end{array}$ \\
\hline Chile & Pública & Media & Se mantiene & Doctorado & Sí & Investigación/docencia \\
Brasil & Privada & Media & Disminuye & Doctorado & Sí & Investigación/docencia \\
México & Pública & Novel & Disminuye & Magíster & Sí & Investigación/docencia \\
Argentina & Privada & Senior & Aumenta & Doctorado & Sí & Investigación/docencia \\
\hline $\begin{array}{l}\text { Uruguay } \\
\text { Venezuela }\end{array}$ & Pública & Media & Se mantiene & Magíster & Sí & Gestión/docencia \\
\hline Colombia & Privada & Novel & Disminuye & Magíster & Sí & Investigación/docencia/gestión \\
\hline
\end{tabular}

A través del análisis del discurso (Ibáñez, 1979), tratando el corpus como un caso (Stake, 1999), pudimos determinar, de manera emergente, dos grandes categorías que dan cuenta de la experiencia de las profesoras de Derecho: la primera se refiere al contexto de pandemia y su impacto en su quehacer; la segunda a las brechas que se producen en la carrera académica y a situaciones de discriminación.

$\mathrm{Al}$ preguntarles por la manera en que experimentaron la pandemia, establecimos que, en todos los países, las condiciones estructurales de desigualdad configuraron las situaciones particulares de cada institución y la experiencia personal. La pandemia avanzó sincrónicamente durante la segunda y tercera semana de marzo en Venezuela, Argentina, Uruguay, Brasil, Chile, Colombia y México. De hecho, sobre la situación en Argentina se menciona lo siguiente:

Primero se interrumpieron las clases en la primaria y luego sabíamos que esto era misterioso, que iba a ser muy rápido. El aislamiento se decretó, se extendió mucho y la respuesta de toda la población fue notoriamente masiva [...] cumpliendo con mucho miedo (entrevistada 2).

En la experiencia venezolana, la situación fue similar:

Los dos primeros casos oficiales de Covid-19 fueron hechos públicos el 13 de marzo de 2020 y uno de ellos estaba relacionado con un colegio que queda en el mismo terreno de la universidad donde trabajo, o sea, además nos pegó muy de cerca (entrevistada 3 ).

En Brasil, la profesora comenzó el proceso estando contagiada: «Mi mamá estaba enferma y no podía subir la escalera, no se podía bañar [...] yo también estaba enferma y las dos pensábamos en sobrevivir» (entrevistada 1). 
La continuidad de la docencia fue clave en el inicio de la pandemia. Todas las universidades buscaron la manera de seguir adelante, ya fuera al inicio de las clases, en la mitad del proceso o finalizando. No estaba claro quiénes estaban enfermos, quienes podrían enfermar, pero lo importante era seguir adelante en el proceso formativo. De hecho, la profesora chilena relata: «Las autoridades de la facultad contrataron la plataforma Zoom y a las tres semanas ya estábamos impartiendo docencia remota de emergencia» (entrevistada 6).

La profesora argentina explica: «Lo atamos con alambre. Cuando no sabíamos en qué plataforma ir, la verdad es que nos comunicamos en el último encuentro presencial con los alumnos y dijimos «armamos un grupo de WhatsApp», y organizaron todo como pudieron» (entrevistada 2).

Por lo tanto, el proceso se pudo implementar ante la decisión institucional de continuar con la docencia por la proactividad de las profesoras y la participación de los estudiantes. Sin embargo, para el alumnado también fue compleja la situación.

Una experiencia diferente tuvo la profesora uruguaya que, en ese entonces, era la encargada de gestionar la docencia en su facultad. Relata lo siguiente al referirse al momento en que se enteró del inició de la pandemia: «Mi primer pensamiento, extrañamente, no tan extrañamente, tuvo que ver con la universidad. Me acuerdo de que le dije a la persona con la que estaba: «Se me acaba de caer la facultad encima»» (entrevistada 5). Así, se ha encargado de «conectar en línea a prácticamente 700 colegas con sus cursos y equipos docentes respectivos. Era muy consciente, en ese momento, de lo que implicaba la función de ese trabajo» (entrevistada 5).

En el contexto latinoamericano de desigualdad, las carreras de Derecho continuaron con la docencia como pudieron y si es que tenían los recursos para hacerlo. Algunas capacitaron inmediatamente a los docentes, pero otras no tenían recursos para enfrentar la situación. De hecho, en Venezuela, las universidades públicas no han podido seguir con regularidad la docencia: «La Escuela de Derecho de la Universidad Central de Venezuela tiene un año sin ver clases, o sea, todo el tiempo, desde el 13 de marzo de 2020 hasta ahorita no han tenido clases» (entrevistada 3 ).

En México, hay universidades que utilizan la plataforma Zoom no pagada: « De hecho, la semana pasada, un doctor que da clases [...] me invitó a una evaluación de sus alumnos, y lo mismo, a los cuarenta minutos se cortó la clase» (entrevistada 4).

La segunda categoría se refiere a situaciones de discriminación y brechas que se producen en la carrera académica de las profesoras. Al respecto, todas las profesoras afirmaron haber experimentado, durante su carrera académica, situaciones de discriminación de género.

También confirmaron que, en todas las universidades, hay oficinas de género y reglamentos que tienen como propósito una mayor equidad en la carrera académica. 
Las profesoras noveles relatan que el argumento de los profesores con cargos directivos es que todavía son muy jóvenes:

Desde mi perspectiva, no he tenido las mismas posibilidades que mis colegas y sobre todo que mis colegas hombres [...] yo fui como un tanto discriminada en la universidad $[\ldots]$ porque, literalmente, me decían los directivos: «estás muy joven, tienes cara de niña»» (entrevistada 4 ).

La profesora relata que a los hombres de su edad no les pusieron problemas en ingresar a la academia:

Entonces, sí, muchos colegas, por el simple hecho de ser hombres, tuvieron acceso muy rápido a clases, o sea, de que les dieran esta oportunidad como maestros muy muy rápido, sin hacer, digamos, alguna carrera previa, como la que siento que yo hice (entrevistada 4).

Las profesoras que están en la mitad de la carrera también dicen haber sido discriminadas. En este momento de la formación es donde se encuentran con el «techo de cristal»:

Daré un ejemplo sin dar nombres, ni espacios. Si se presentan dos personas, un hombre y una mujer [...] para concursar, si es mujer dicen que se va a distraer con su familia y las cosas propias de ser mujer, entonces, pese a tener la mayor condición [...] gana el hombre [...] y, bueno, una dice que no es posible, que no es justo, que no corresponde (entrevistada 2).

En esta etapa de la formación, también se encuentran con la «frontera de cristal», sintiendo que sus esfuerzos no han dado todos los frutos esperados. Para seguir la carrera académica, la profesora brasilera cuenta que debió postergar la maternidad para poder llevar el ritmo de los profesores. De hecho, se exigió hacer las labores académicas mejor que los hombres: «Si yo me olvido no tengo perdón», menciona. Por eso, decidió trabajar más que los hombres y postergar la maternidad. Hoy, a sus 41 años, recién está embarazada. Sobre esto, menciona: «Si hubiese sido hombre, podría haber tenido una familia mucho antes».

La «frontera de cristal» también da cuenta de una tensión entre las labores familiares y la carrera académica, implicando un gran esfuerzo por continuar. Sobre este punto, una de las profesoras menciona: «siempre hubo espacio para mi hijo, para prepararle su desayuno, su lonchera, para acostarlo, para darle su comidita, para ayudarlo a hacer sus tareas, para llevarlo al médico [...] y para mi esposo» (entrevistada 3 ).

De hecho, en su momento, dejó un puesto directivo para estar mas cerca de su hijo: 
En algún momento, cuando mi hijo entró a la adolescencia, yo era la vicerrectora de Investigación de la universidad, pero empecé a notar que él sentía que yo me había alejado un poco. Yo dije: «Señor rector, yo no voy a seguir con la vicerrectoría. Me encanta el cargo, creo que hemos hecho muchas cosas, pero no voy a seguir, primero está mi hijo (entrevistada 3 ).

El cambio se produjo a nivel personal, pero también a nivel cultural. De hecho, en Uruguay, la profesora entrevistada menciona que, en su experiencia, en la universidad operan iguales oportunidades entre hombres y mujeres: «En lo que a mí corresponde, el instituto mío, de origen en general, ha tenido participación tanto de hombres como de mujeres» (entrevistada 5).

En la etapa senior, las profesoras también dicen haber sufrido discriminación de género:

Sí, como mujer ha sido difícil conseguir lo que he tenido, ha habido desconfianza, ha habido maltrato, ha habido rechazo, no sé por qué generalmente me encuentro en escenarios donde hay cinco hombres y yo soy la representación femenina (entrevistada 3$)$.

La carrera académica de la profesora fue hecha en un contexto masculinizado, en que se sale adelante sin que la institución genere espacios de equidad para desarrollarla con las mismas oportunidades.

La profesora chilena también pasó por renuncias para avanzar en la carrera académica: «Recuerdo que, mientras estaba terminando el doctorado, tenía que cuidar a mis hijos, trabajaba en la universidad, y en la noche no dormía para avanzar en mi tesis. Fueron años muy difíciles» (entrevistada 6).

En esta etapa, este sacrificio también lo experimenta una de las profesoras noveles, quien afirma:

Yo agradezco que usted se tome el tiempo de preguntar qué está pasando con las mujeres profesoras de Derecho. Mi salud se ha visto muy afectada. Para que me dé tiempo de todo, hago algo desde hace muchos años: yo le llamo «tercer turno», ¿no? En el primer turno, en el de la mañana, doy las clases, hago mis planeaciones, leo cuestiones de la investigación, todo lo que tiene ver con mi vida académica [...] el segundo turno, como yo lo llamo, es en las tardes, empieza con la hora de la comida: estar con mi hija, hacer actividades, de pronto sus tareas [...] el tercer turno ocurre cuando mi hija ya duerme, cuando ella está descansando y todo [...] me pongo a trabajar y hago mis avances de tesis, prácticamente mis avances han sido en la madrugada (entrevistada 4).

Creemos que este trabajo de tres turnos en tiempos de pandemia debe representar la situación en la que viven muchas profesoras en sus carreras académicas. Queda mucho camino por recorrer. 


\section{Referencias}

Baeza Reyes, Andrea y Silvia Lamadrid Álvarez (2019). «¿Igualdad en la academia? Barreras de género e iniciativas en una universidad pública (2013-2018).»Pensamiento Educativo, Revista de Investigación Latinoamericana (PEL), 56 (1): 1-17. DOI: 10.7764/PEL.56.1.2019.9.

BURIN, Mabel (2008). «Las «fronteras de cristal» en la carrera laboral de las mujeres. Género, subjetividad y globalización». Anuario de psicología/The UB Journal of psychology, 39 (1): 75-86. Disponible en bit.ly/3h6DZOZ.

Cepal, Comisión Económica para América Latina y el Caribe (2020). La economía del cuidado como acelerador el cambio estructural con igualdad. Disponible en bit. ly/3h3NQFd.

Díaz Martínez, Capitolina (2020). «Obstáculos para la igualdad de género en las universidades».Rued@. Revista Universidad, Ética y Derechos, 1 (5): 60-76. Disponible en bit.ly/3dtcHzR.

Fardella Cisternas, Carla, Alejandra Corvalán-Navia, Javiera García-Meneses, y Francesca Chiappini Koscina (2021). «Ni extranjeras, ni secretarias: Discursos de las científicas chilenas sobre el trabajo académico». Pensamiento educativo, 58 (1), 1-13. DOI: 10.7764/PEL.58.1.2021.11.

Flores-Sequera, Mitzy Magaly (2020). «Desigualdades develadas por la pandemia: Economía del cuidado y malestar en profesoras de universidades venezolanas». Antropología Americana, 5 (10) : 95-111. Disponible en bit.ly/3dot6Wb.

García-González, Mariluz, Fermín Torrano y Guillermo García-González (2020). «Estudio de los factores de riesgo psicosocial en profesoras de universidades online: Una mirada desde adentro». Interdisciplinaria. Revista de Psicología y Ciencias Afines, 37 (1): 275-292. DOI: 10.16888/interd.2020.37.1.18.

IвAÑEZ, Jesús (1979). «Interpretación y análisis del discurso». En Más allá de la sociología. El Grupo de Discusión, técnica y crítica (pp. 333-351). Madrid: Siglo XXI.

LARA, Catalina (2007). «La perspectiva de género en los sistemas de evaluación de la producción científica». Revista de Investigación Educativa, 25 (1): 133-148. Disponible en bit.ly/35XHP6u.

Lozano Cabezas, Inés, Marcos Jesús Iglesias Martínez y María Ángeles Martínez Ruiz (2016). «Un estudio cualitativo sobre los diferenciales de género en la educación superior: Percepciones de las académicas en contextos masculinizados». Revista la manzana de la discordia, 11 (1):41-54. DOI: 10.25100/lamanzanadeladiscordia.v11i1.1633

PNUD, Programa de las Naciones Unidas para el desarrollo (2021). Atrapados: Alta desigualdad y bajo crecimiento en América Latina y el Caribe. Informe Regional de Desarrollo Humano 2021. Disponible en bit.ly/2USaVlB. 
SAID-Hung, Elias, Beatriz Marcano y Rebeca Garzón Clemente (2021). «Ansiedad académica en docentes y Covid-19. Caso instituciones de educación superior en Iberoamérica». Revista Prisma social, Revista de Ciencias Sociales, 33 (2): 289-305. Disponible en bit.ly/3joHul8.

Santos Pérez, Antonia, Carolina Garcés Estrada y Loreto Castillo Collado (2020). "Usted, chiquilla, también puede'. Reflexiones en torno al sexismo en la universidad en estudiantes de Derecho». Revista Pedagogía Universitaria y Didáctica del Derecho, 7 (2), 119-142. DOI: 10.5354/0719-5885.2020.57746.

STAKE, Robert (1999). Investigación con estudio de caso. Madrid: Morata.

TAYLOR, Steve, Robert Bogdan (1984). Introducción a los métodos cualitativos de investigación. Barcelona: Paidós.

\section{Sobre la autora}

María Francisca Elgueta es doctora en Educación por la Universidad de Valladolid y directora de la Unidad de Pedagogía Universitaria y Didáctica del Derecho de la Facultad de Derecho de la Universidad de Chile. Su correo electrónico es cpudd2@ derecho.uchile.cl. (D) https://orcid.org/oooo-0002-4212-3960. 
La Revista Pedagogía Universitaria y Didáctica del Derecho (RPUDD) es una publicación científica semestral que contribuye a la reflexión multidisciplinaria sobre pedagogía universitaria y didáctica del derecho, para la formación y consolidación de esta área de investigación; así como a la difusión de prácticas innovadoras en la enseñanza-aprendizaje del derecho considerando el contexto nacional e internacional. Es una publicación electrónica internacional con una codirección entre Brasil y Chile.

\author{
DIRECTORA \\ María Francisca Elgueta Rosas \\ Universidad de Chile \\ DIRECTOR \\ Renato Duro Dias \\ Universidad Federal de Rio Grande, Brasil \\ SITIO WEB \\ pedagogiaderecho.uchile.cl \\ CORREO ELECTRÓNICO \\ rpedagogia@derecho.uchile.cl \\ LICENCIA DE ESTE ARTÍCULO
}

Creative Commons Atribución Compartir Igual 4.o Internacional

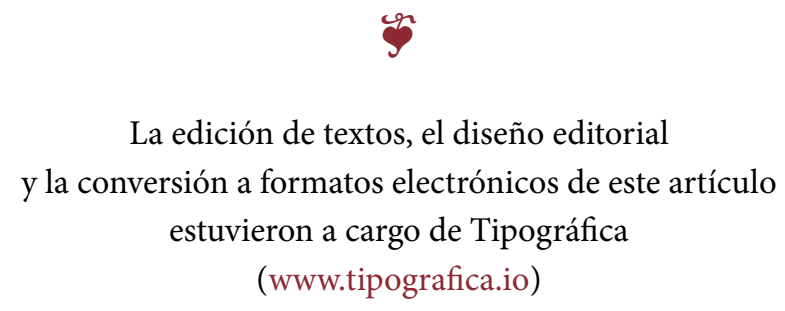

